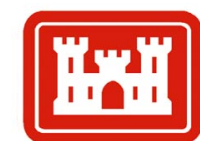

US Army Corps of Engineers

\title{
Representation of Hydrodynamic Model Results through Graphical Displays
}

\author{
by Mark S. Gosselin, R. Bruce Taylor, and Kenneth R. Craig
}

PURPOSE: The Coastal Engineering Technical Note (CETN) described herein contains information and procedures for displaying hydrodynamic modeling results within the Surfacewater Modeling System (SMS) platform. Such visualization facilitates the interpretation of large amounts of complex output generated by the models, as well as assist the engineer in communicating modeling results to laymen, planners, and others who lack expertise.

BACKGROUND: With the increasing application of numerical modeling methods by practicing professionals, attention has shifted to enhancing the capabilities for displaying model output. Methods developed by the Diagnostic Modeling System (DMS) (Kraus 2000) that aid in interpreting physical flow features and coupling of hydrodynamics with sediment transport receive special emphasis in this CETN. Traditional visualization techniques are reviewed, followed by more modern products that have proved their utility in support of engineering projects.

Traditional output formats for displaying hydraulic model results were mostly limited to black and white flow vector plots and time-histories of water-surface elevation, current, or flow rate discharge at selected locations of interest. Figures 1 through 3 show examples of these types of output. Although helpful, they do not provide adequate insight on the flow or sediment movement. Flow vector plots (Figure 1), although depicting representative flow patterns of the entire system, are qualitative and heuristic. They leave the engineer with no direct means of distinguishing problematic flow conditions from acceptable conditions. Similarly, time-history plots, although specific and quantitative, convey no relationship between data depicted at one location to the overall behavior of the system. Recent attention to graphical display of model results has addressed these shortcomings.

With the advancement of computer technology and graphics capabilities, more varied and creative ways for displaying model output have begun to appear. Figures 4 through 6 show examples, all of which represent significant improvements in diagnostic output formats. In Figure 4, overlaying the velocity vectors on a false color background of the modeled bathymetry enhances the traditional flow vector plot. This output format gives the user insight into the broad flow properties of the system and how they are controlled by bathymetry, a significant diagnostic relationship. Figure 5 displays quantitative velocity information in a format significantly different than the traditional time-history plots shown in Figures 2 and 3. Here, values of velocity, computed at each mesh element, generate isovels or contours of equal velocity. By displaying various parameters (e.g., bathymetry, velocity) together, the user can understand the extent of specific thresholds of strong and weak currents and the relationships of these areas to shoreline orientation, structures, and bathymetry. 


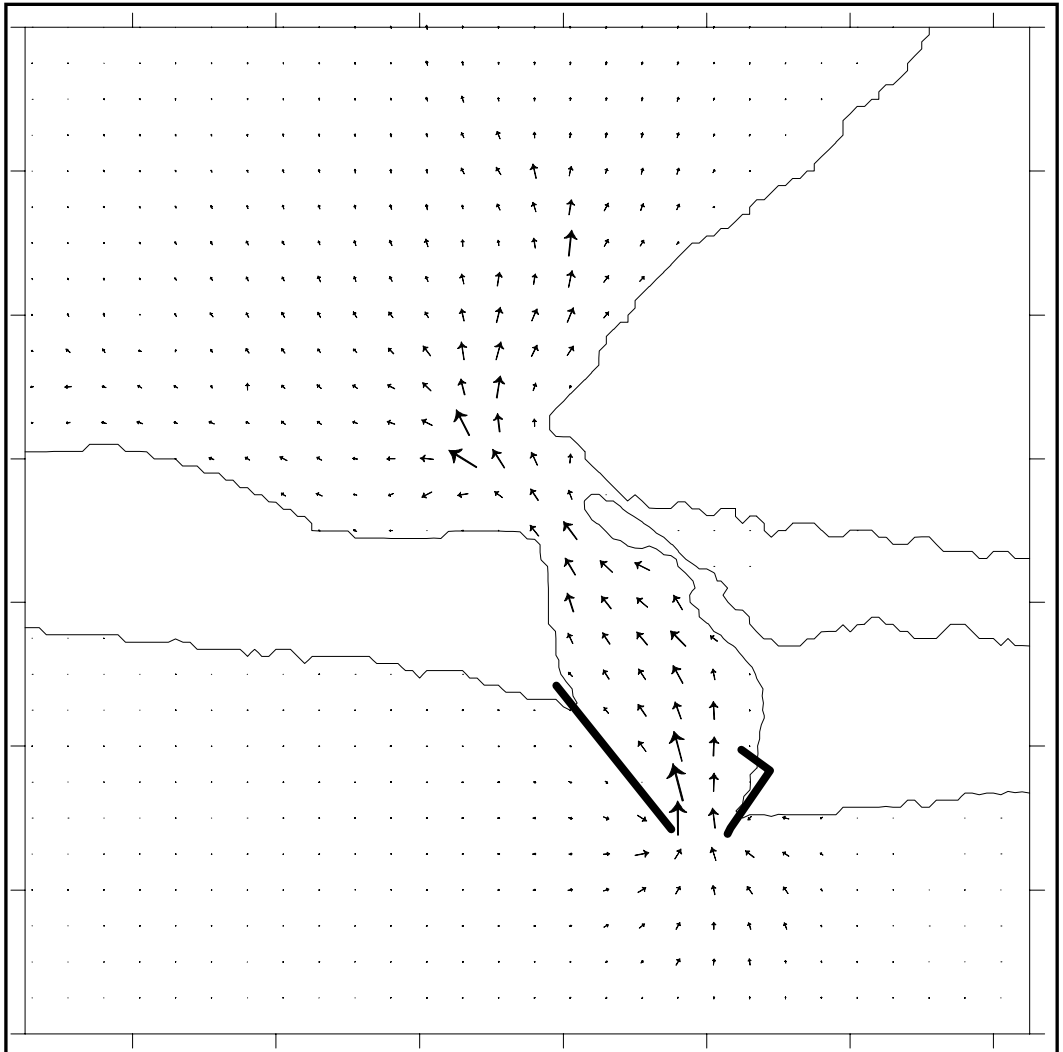

Figure 1. A traditional black and white velocity vector plot that provides a qualitative picture of the entire system, but not specific causal relationships between flows and bathymetry

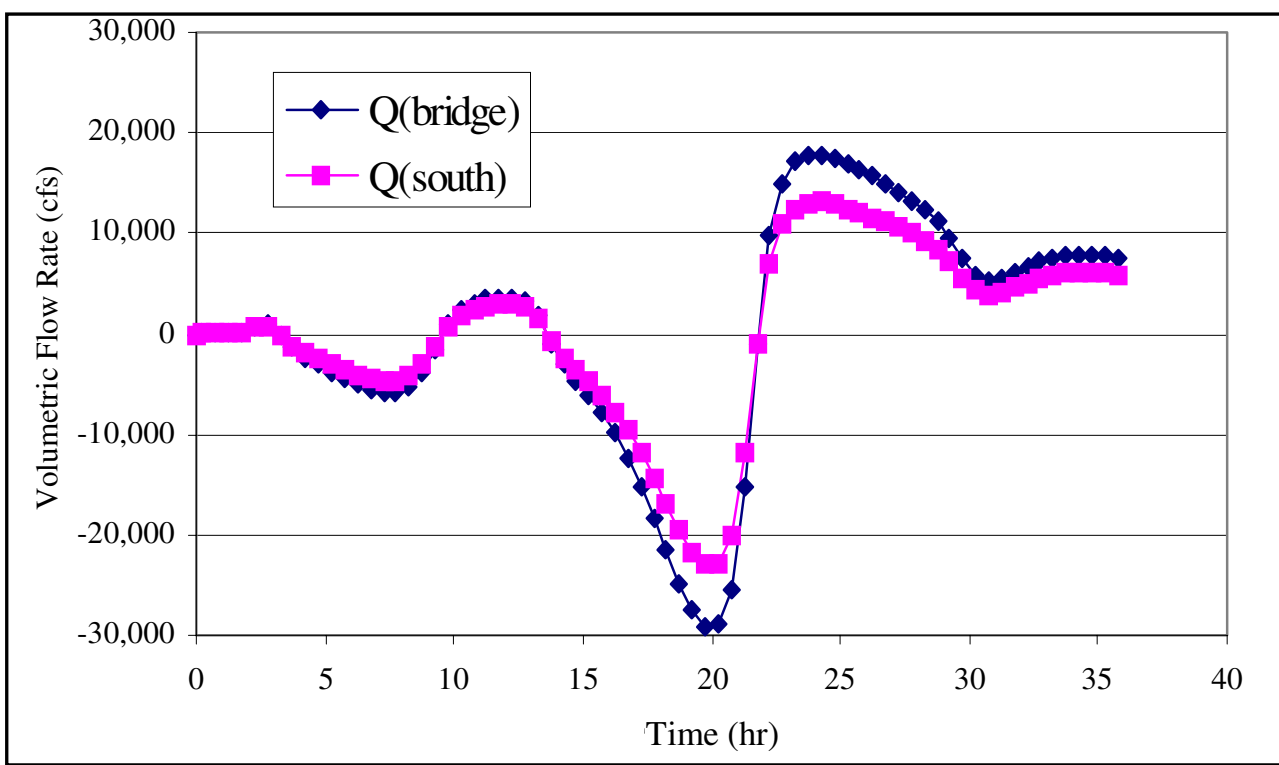

Figure 2. A traditional discharge time-history plot that provides point-specific, quantitative information, but fails at providing a picture of the flow conditions over the entire domain (Volumetric flow rate is in cubic feet per second. To convert to cubic meters per second, multiply by 0.3048 ) 


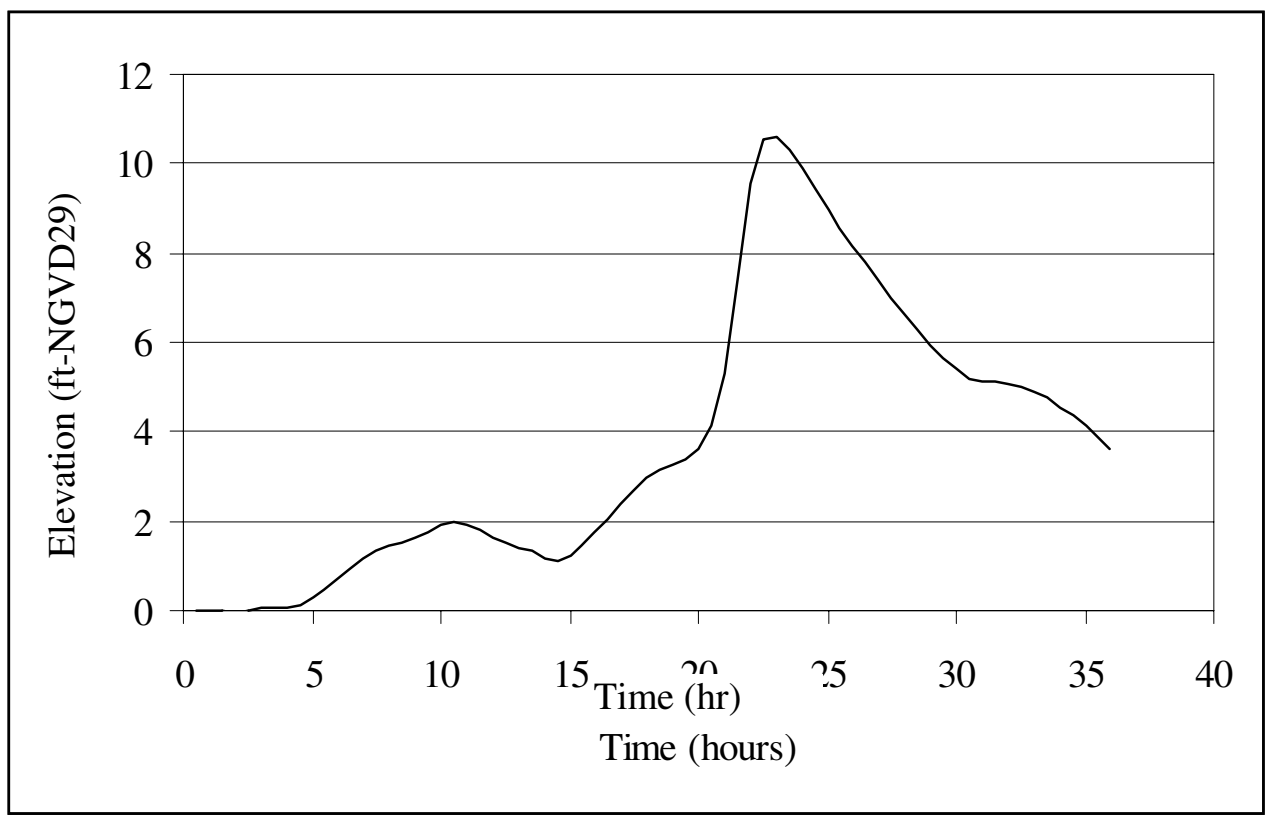

Figure 3. A traditional elevation time-history plot that provides point-specific, quantitative information, but does not give a picture of the flow conditions over the entire domain ${ }^{1}$

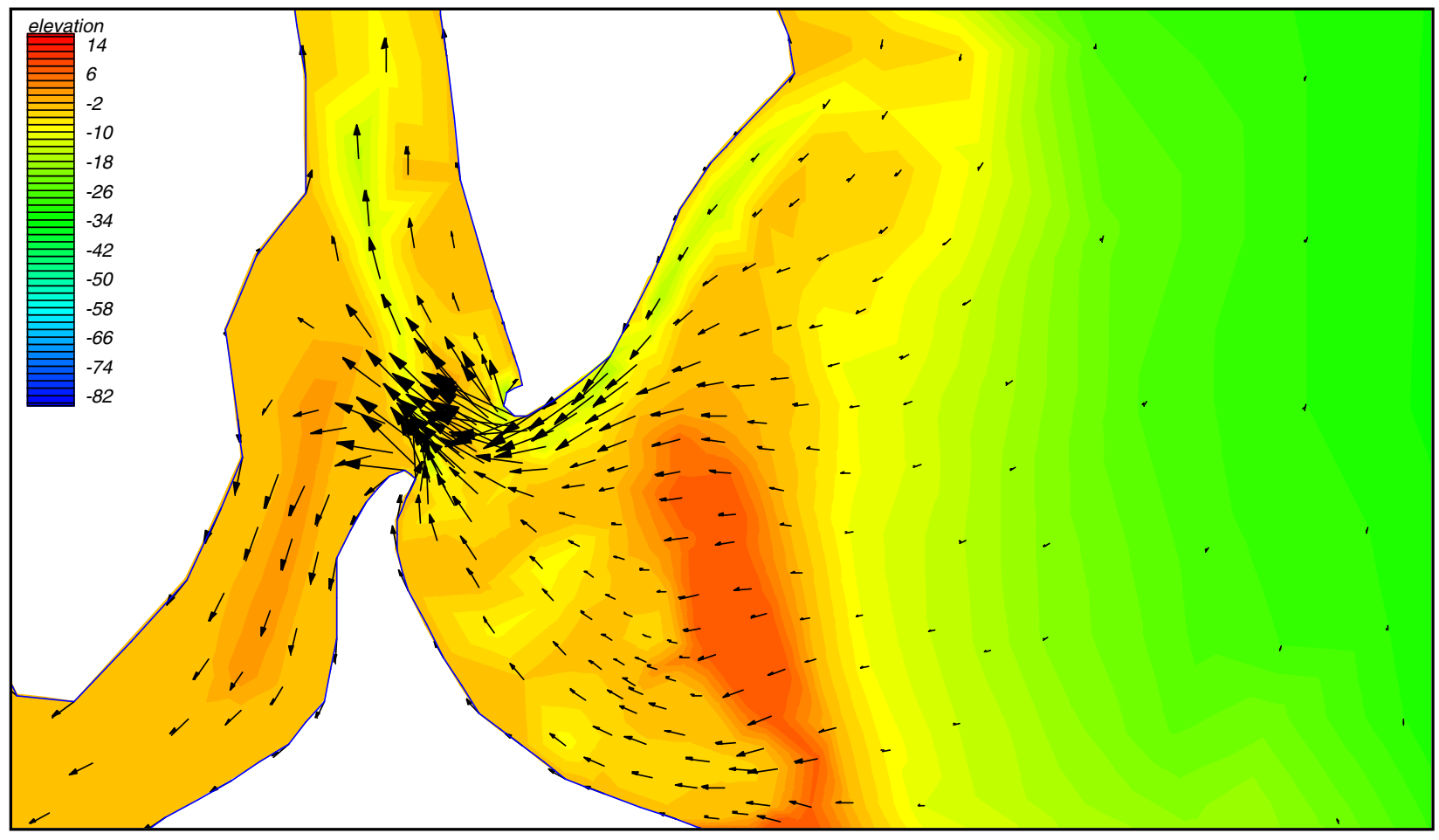

Figure 4. A false-color bathymetry filled contour plot with velocity vector overlay plot (units in $\mathrm{ft}$ ) that illustrates causal relationships between velocity and bathymetry

${ }^{1}$ All elevations (el) cited herein are in feet referenced to the National Geodetic Vertical Datum (NGVD) (To convert feet to meters, multiply by 0.3048). 


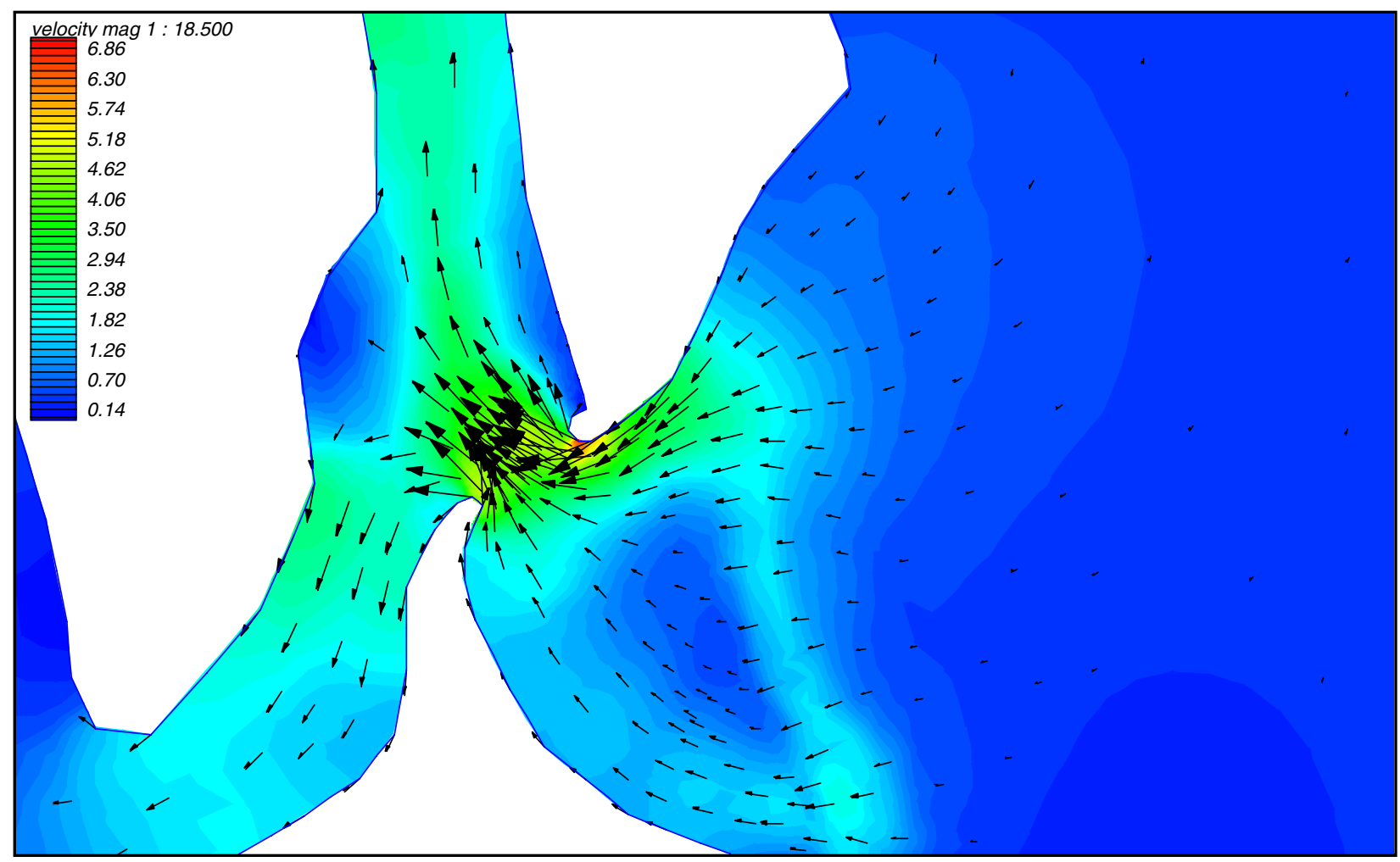

Figure 5. An isovel-filled contour plot with velocity vector overlay (units in $\mathrm{ft} / \mathrm{s}$ ) that provides quantitative information over the entire area of interest (Velocity is in feet per second. To convert to meters per second, multiply by 0.3048 )

Finally, in Figure 6, the logic behind Figure 5 extends one step further. In this figure, contours of change in velocity are shown with increased velocities in red and decreased velocities in blue. These types of formats are particularly helpful for the evaluation of proposed or expected changes in bathymetry, channel alignment, or structural modifications. To generate this type of plot, the hydrodynamic model must be run twice - once for baseline conditions and once for the changed conditions under consideration. Computed velocity values for each model simulation are then overlaid to create a single new output file representing the change in velocity at each element. These data are then displayed, as in the contours shown in Figure 6. The color contours readily highlight those areas which may become prone to erosion (red) and deposition (blue) should the condition change. This CETN provides guidelines for creating plots from hydrodynamic output, such as Figures 4 through 6, that improve the transfer of flow information, enable an improved level of understanding about the model results, and provide insight into sediment transport without having to run a time-dependent sediment transport model.

Although this CETN functions together with the DMS as a guide for identifying areas of persistent shoaling through graphical interpretation, the methods described herein are applicable for displaying hydrodynamic output of any kind, including those obtained from physical models. This CETN first reviews the platform (SMS) (Brigham Young University 1999) for creating the types of plot presented in Figures 4 through 6, and then discusses presentation of the direct hydrodynamic output. Finally, the discussion ends with methods to manipulate the output to gain further insight into the flow physics and sediment transport. 


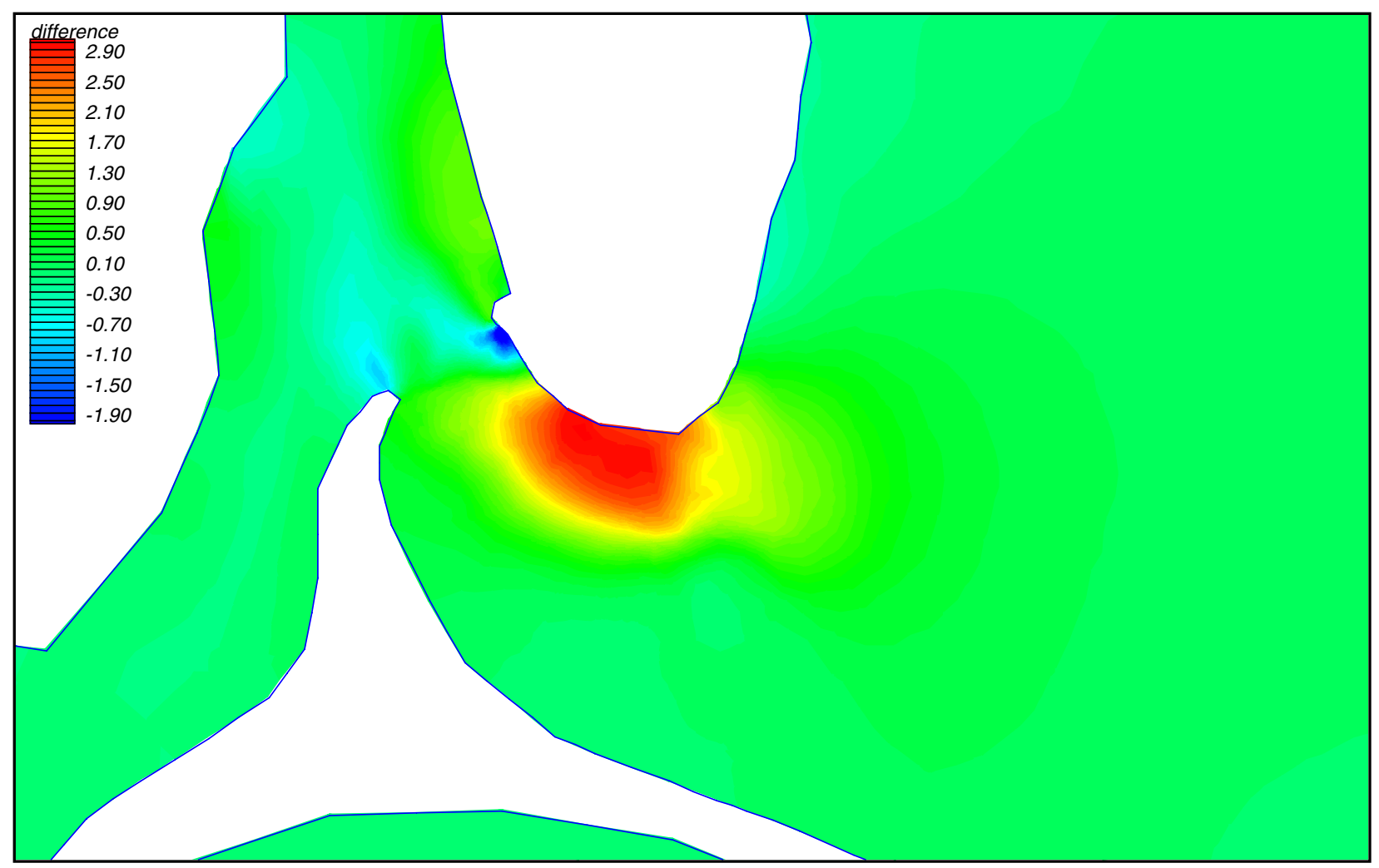

Figure 6. A filled contour plot of changes in velocity (units in $\mathrm{ft} / \mathrm{s}$ ) that illustrates cause and effect on velocity fields associated with engineered modifications to an inlet (To convert feet per second to meters per second, multiply by 0.3048 )

SURFACEWATER MODELING SYSTEM (SMS) FUNCTIONALITY: The SMS was developed by the Engineering Computer Graphics Laboratory at Brigham Young University (BYU) under sponsorship by the U.S. Army Corps of Engineers and the U.S. Federal Highway Administration (FHWA). The SMS is a pre- and post-processor for numerous hydrodynamic modeling programs including ADCIRC, FESWMS, HIVEL2D, RMA2, RMA4, and WSPRO. It is a pre- and post-processor for surface-water modeling and analysis (BYU 1999). Further information about the SMS can be located at the following address: http://chl.wes.army.mil/software/sms/. This CETN discusses only the recent (Version 7) postprocessing capability of SMS to produce an array of contour and velocity vector plots from hydrodynamic model results. In addition, the SMS Data Calculator feature is discussed for creating data sets that aid in discerning flow and sediment transport behavior.

The SMS contains the capability for creating contour plots of scalar data on a finite-element mesh. Three different types of contours are available, including normal linear contours, color fill between contours, and cubic spline contours. The user controls the number of contours, the contouring interval, minimum and maximum contour values, labeling options, bolding contours, and the color scale. SMS also can produce vector plots from vector data on a finite element mesh. The user controls the arrowhead style and size, vector length, vector placement and density, and color. The user can also vary the vector length and vector color proportionately with the velocity magnitude. 
In addition to its plot-making abilities, the SMS can construct vector data sets from scalar data, and vice versa. Also, the data calculator allows the user to manipulate multiple scalar data sets with several simple operations to form new scalar data sets. A subsequent section presents an example of such a manipulation.

PRESENTATION OF OUTPUT: Default output from two-dimensional, depth-averaged, hydrodynamic models includes water-surface elevation and velocities - velocity magnitude as a scalar data set and speed and direction as a vector data set. In addition, model input contains a bathymetry data set. The drawing of causal relationships between the bathymetry and the speed and direction is the key to understanding simulation results. Discussion will concentrate on the creating model output plots that better illustrate these casual relationships and increase understanding of the acting processes. For all subsequent figures, the example plots were created from a simulation of the flow at East Pass, FL, during spring ebb tide.

A standard vector plot with an outline of the shoreline is shown in Figure 1. As shown in Figure 7, vector plots are greatly enhanced when overlaid on a false color bathymetry plot. The contour range is set at the limits of the bathymetry bounded by the plot rather than the entire mesh to show greater detail. In addition, the number of contours has been increased from the default number of 10 to 40 to show even more contrast. The legend on the left hand side has been expanded from the default length, as shown in Figures 4 through 6, to span the entire height of the plot. This expansion facilitates contour identification in working with a greater number of contours. The vector overlay plot includes setting the vector length proportional to the vector magnitude. From this plot, the influence and control exerted by the local water depths are readily seen. For example, the velocities decrease following the vertical expansion over the ebb shoal. Also, the velocities north of the spur jetty show a marked decrease, indicating that the spur functions in its capacity to deflect high velocity flows away from the shoreline north of the east jetty. Beyond these observations, distinguishing the flow behavior in the channel is difficult given that the eye cannot discern the small differences in the vector lengths as water moves from north to south. Another difficulty associated with this plot is discerning the flow patterns in areas of small finite elements (e.g., between the jetties). To address this difficulty, SMS contains the capability to create a grid of vectors at a user defined spacing rather than attach the vectors to each node. Figure 8 illustrates this capability. From this figure, one can readily discern the flow patterns between the jetties as opposed to Figure 7 where no patterns are discernable. This technique must be applied judiciously, however. Too large a grid spacing may not resolve the flow features that the modeler was trying to capture with the small elements in the first place. 


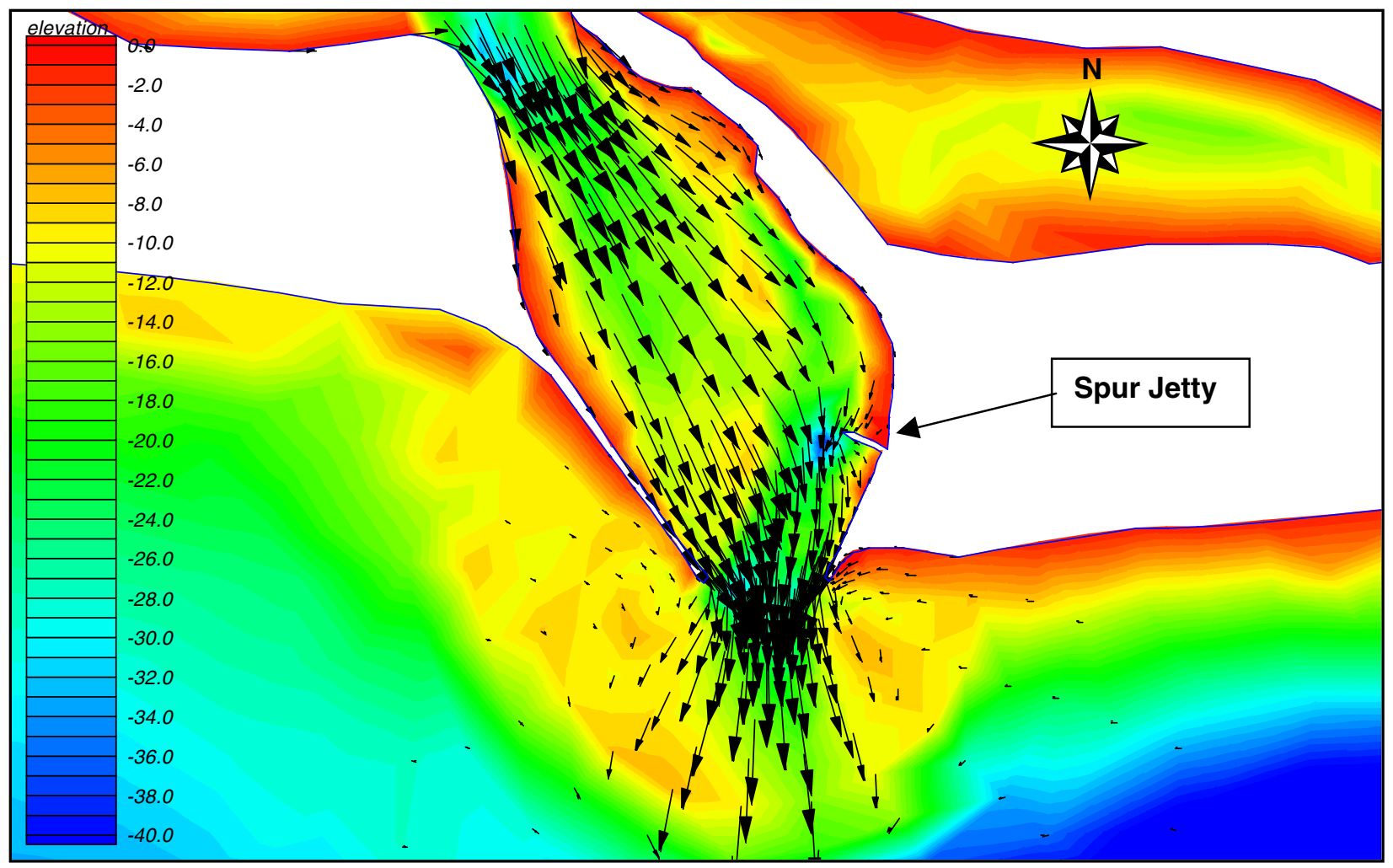

Figure 7. Velocity vector plot overlaid on contours of bathymetry (units in $\mathrm{ft}$ ) (To convert feet to meters, multiply by 0.3048 )

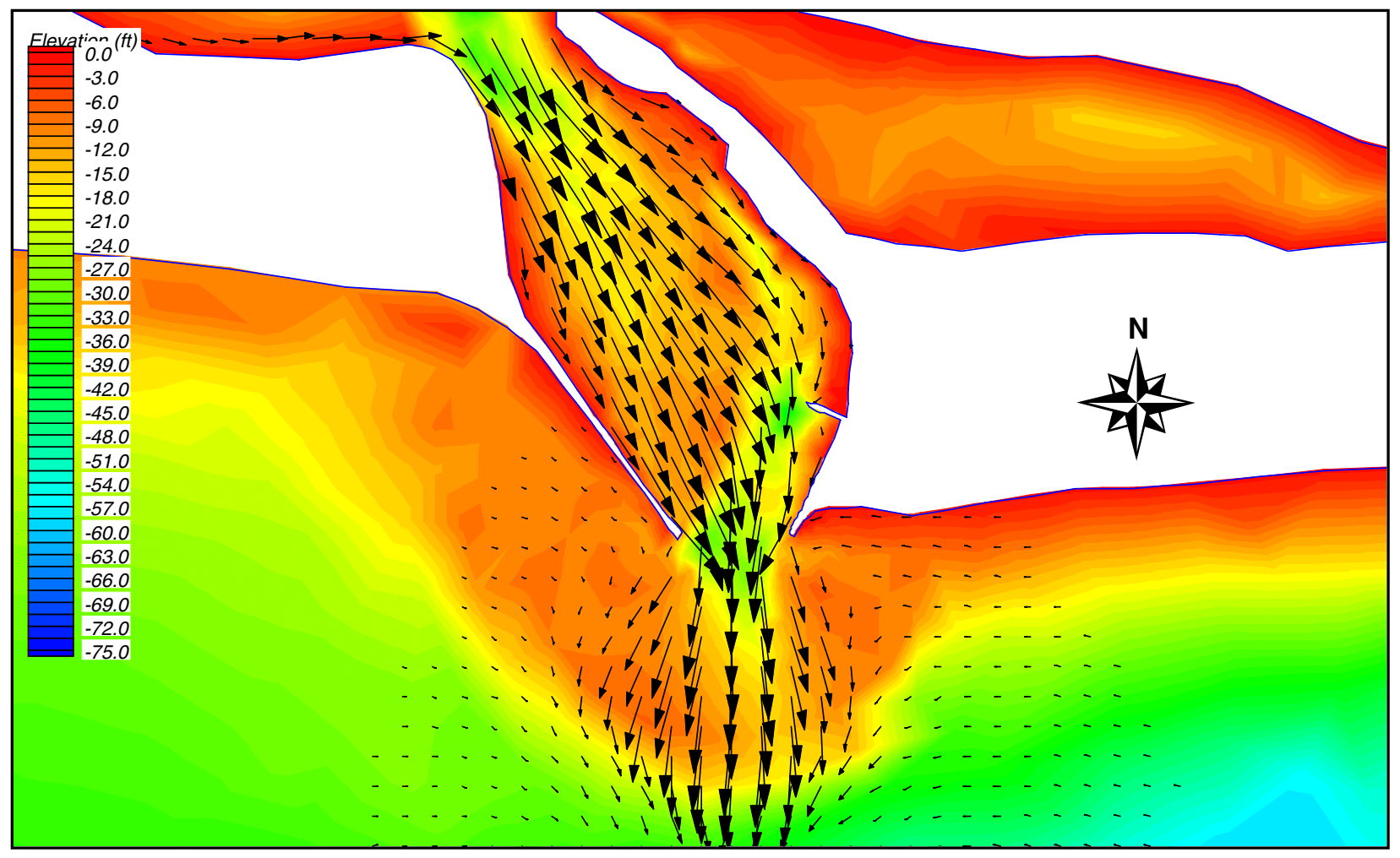

Figure 8 . Gridded velocity vector plot overlaid on contours of bathymetry (units in $\mathrm{ft}$ ) (To convert feet to meters, multiply by 0.3048 ) 
To better comprehend the velocity variation within the channel, Figure 9 illustrates a vector plot overlaid on a contour plot of velocity magnitude. The contour plot shows many of the same techniques discussed with the previous figure. The contour plot employs the psychological association of the cooler (blue) colors with inactivity (lower velocities) and hot colors (red) with activity (higher velocities) by reversing the default color scale. The vector lengths were rescaled, compared to Figure 7, to display the underlying color contours. Also, the lowest magnitude vectors were set to a finite length to show behavior in regions of low velocity. Finally, the vectors were set to white to provide contrast with the darker contours.

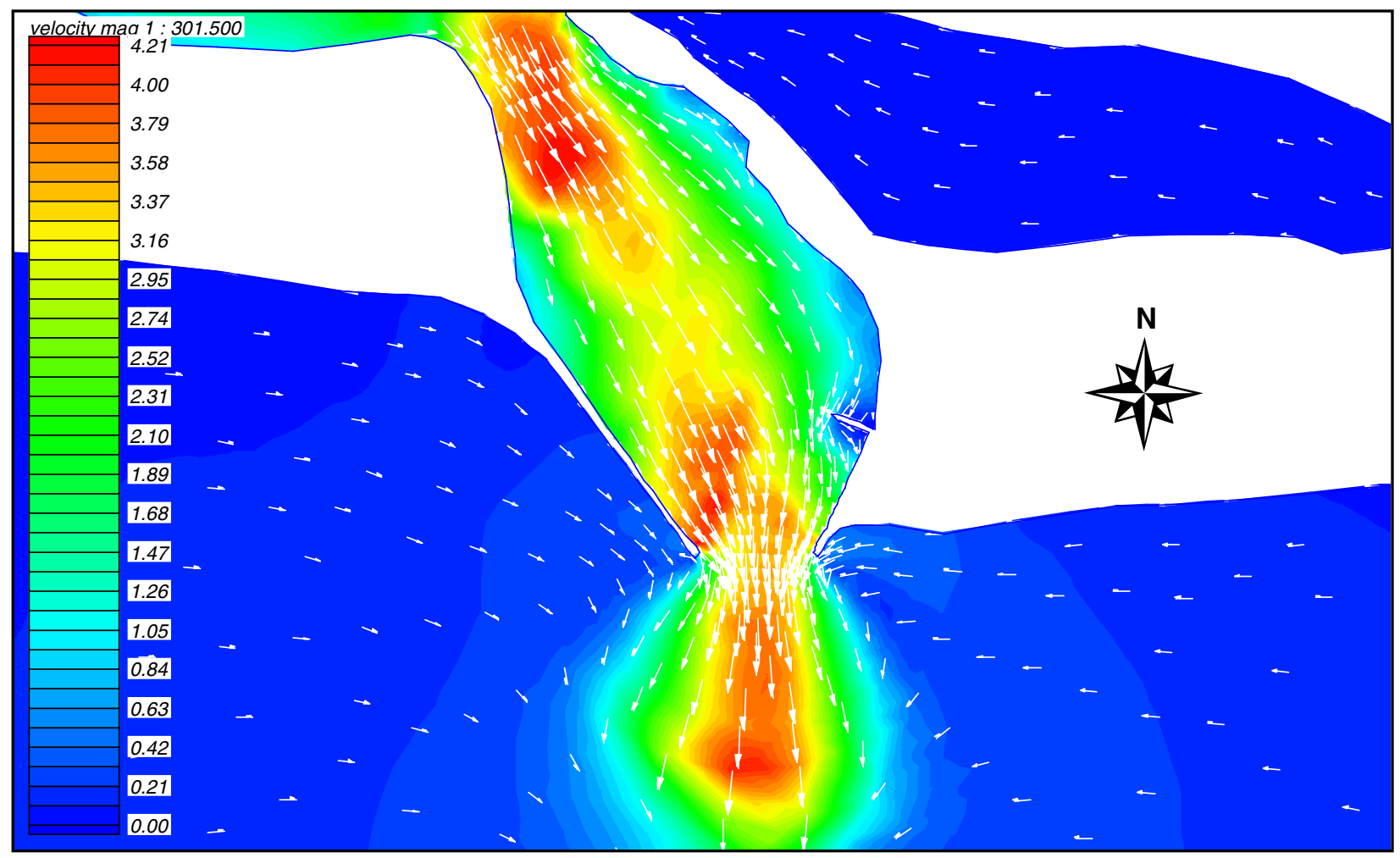

Figure 9. Velocity vector plot overlaid on contours of velocity magnitude (units in $\mathrm{ft} / \mathrm{s}$ ) (To convert feet to meters, multiply by 0.3048 )

Figure 9 gives much more insight into the velocity variation in the channel as compared to Figure 7. Apparent from the figure, a region of acceleration occurs at the north end of the channel just south of the constriction. Next, flow decelerates as it enters the widening channel. Before exiting, the flow accelerates again, but only on the west side of the channel. Another notable feature displayed is the entrainment into the ebb jet that extends along the shoreline on either side. This feature could only be resolved by setting a minimum vector length.

Figure 9, however, has lost the capability for distinguishing the cause-and-effect relationship between the flow and the bathymetry. Figure 10 reintroduces this capability. The figure displays a gray scale contour plot of bathymetry overlaid with a velocity vector plot. The vector length and color are proportional to the velocity magnitude. The velocity vector color scale employs the same variation as the contours in Figure 9. Notably, the eye is directly drawn to the regions of interest in red. By this graphical means, the relationship between the velocity 
magnitude and the bathymetry becomes apparent. For example, the acceleration following the northernmost constriction is due to a broad shoal located on the west side of the channel. Also, the acceleration located on the west side of the channel before the flow exits is attributed to the shallow water on the west side and the deep channel on the east. Figure 10 combines the detailed magnitude information contained in Figure 9 with the bathymetry information contained in Figure 7.

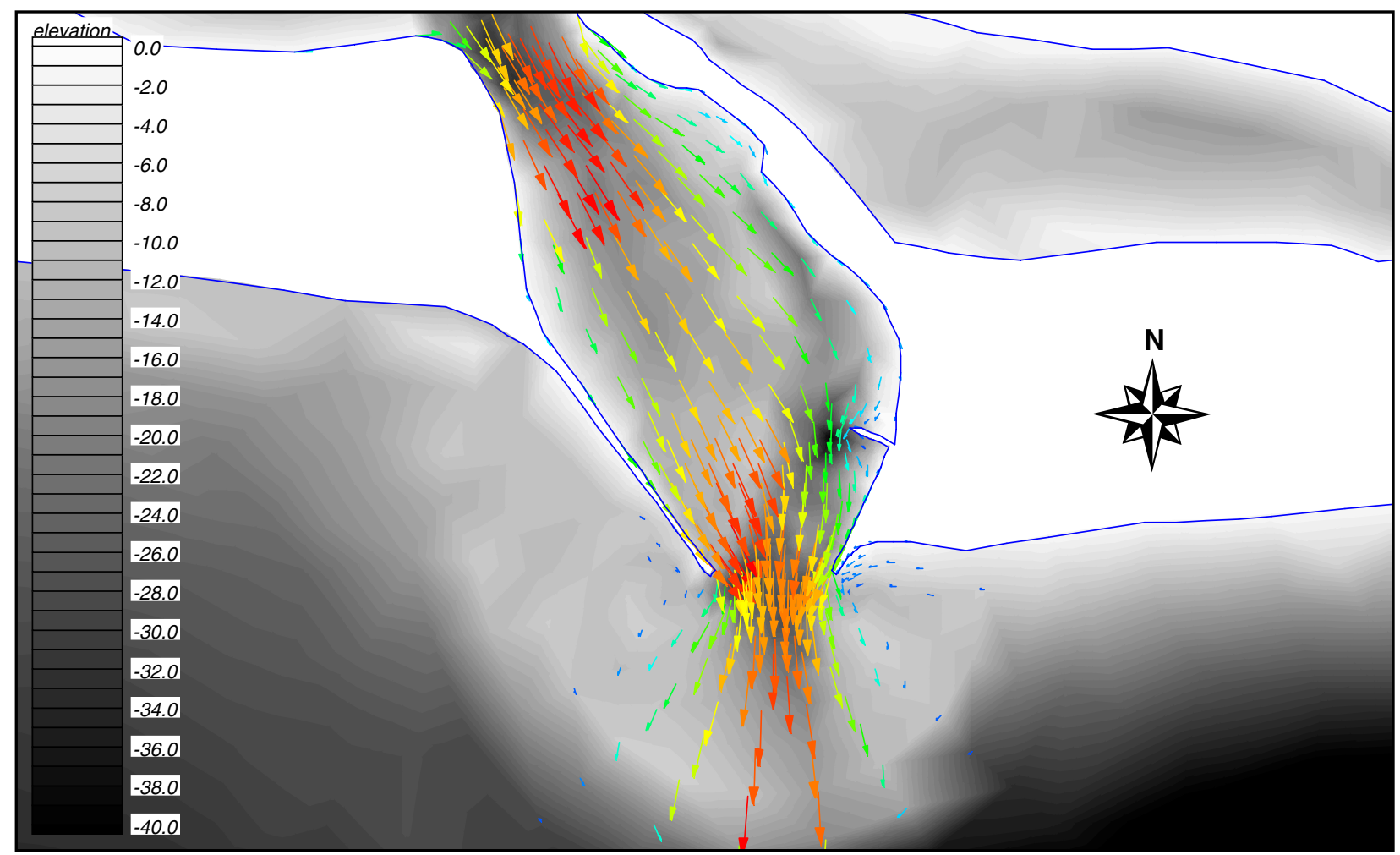

Figure 10. Color velocity vector plot overlaid on contours of gray scale bathymetry (units in $\mathrm{ft}$ ) (To convert feet to meters, multiply by 0.3048 )

MANIPULATION OF OUTPUT: Often, hydrodynamic simulations are performed to evaluate engineered modifications or to evaluate shoaling or scouring trends in a particular area. This section discusses aids that can be developed for arriving at conclusions about these actions by creating plots from the manipulation of the default model output.

\section{Comparison Plots}

Comparison plots allow the modeler to detect differences in solutions from two simulations and thereby evaluate the consequences of project alternatives. To illustrate this capability, flow conditions are compared with alternative spur jetty configurations; serving as the base conditions, the spur is oriented perpendicular to the eastern shore (see Figure 7), whereas the spur is oriented parallel to the shoreline for the alternative configuration. As shown in Figures 7 through 10, the spur deflects the flow towards the center of the inlet; however, a large scour hole has formed at its tip. The alternative, shown in Figure 11, reorients the spur to present less of a flow obstruction to reduce tip scour and still protect the shoreline. 


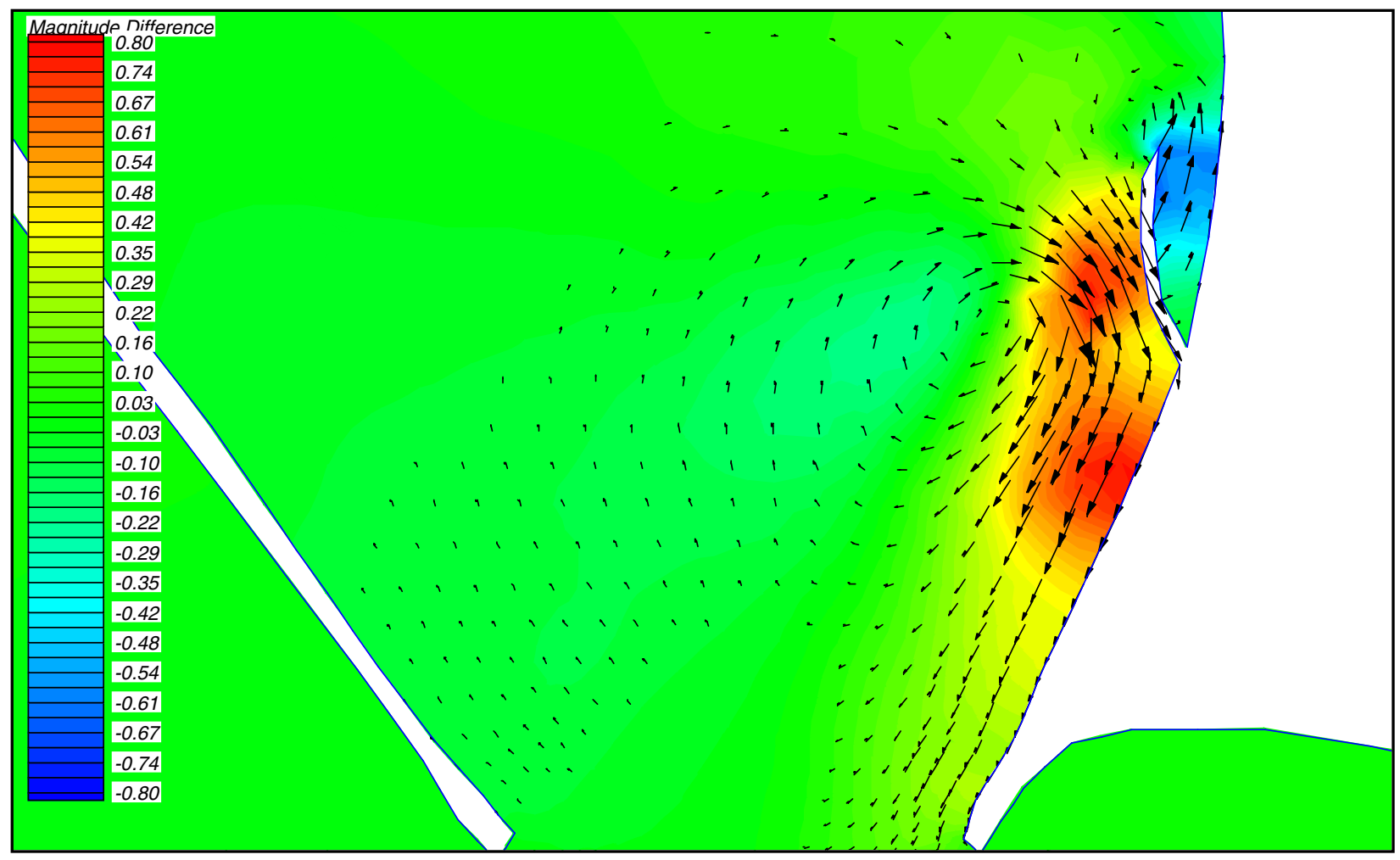

Figure 11. Velocity vector difference plot overlaid on contours of velocity magnitude difference (units in $\mathrm{ft} / \mathrm{s}$ ) to evaluate proposed reorientation of the spur jetty (To convert feet per second to meters per second, multiply by 0.3048 )

Figure 11 shows the effectiveness of the alternative during the same flow conditions shown in previous alternatives. The plot contains contours of velocity magnitude difference overlaid with a plot of velocity vector difference. The plot was constructed by first running the existing baseline condition and the proposed alternative condition. To create the contours, the baseline output was converted to scatterpoint data. This data set was then interpolated onto the alternative condition mesh. Finally, the baseline velocity magnitude scalar data set was subtracted from the alternative velocity magnitude data set via the SMS data calculator to create a velocity-difference data set. The contours show this velocity difference. Positive values indicate increases in velocity associated with the alternative, and negative values indicate decreases. The vector data set was created by first deconstructing the baseline vector data set into its $\mathrm{x}$ - and $\mathrm{y}$-components. These scalar data sets were then converted into scatterpoint data and interpolated onto the alternative mesh. The alternative vector data set was also deconstructed into its $\mathrm{x}-$ and $\mathrm{y}-$ components. Next, two scalar data sets of the difference between the alternative and the baseline $\mathrm{x}$ - and $\mathrm{y}$-velocity components were created via the SMS data calculator. Finally, a vector data set was constructed from the two difference data sets. The result is the subtraction of the baseline vector from the alternative vector. The vector length is proportional to the magnitude of change.

The contours reveal areas of increase and decrease in velocity magnitude. The alternative appears to reduce the velocity magnitude near the shoreline (indicated by the blue contours). However, a marked increase occurs in the velocity just west of the east jetty (indicated by red 
contours). The vector plot shows the direction of the increase or decrease. For example, in the region of velocity magnitude increase just west of the spur, the vectors show the increase directed at the point where the spur meets the east jetty. An increase implies that this alternative may experience an increase in foundation scour in this area - an unintended consequence of this engineering alternative. Clearly, plots of this type can help identify consequences, both positive and negative, caused by modifying existing configurations.

\section{Sediment Transport}

Often, hydrodynamic simulations are performed to gain insight into scour and deposition patterns for a particular area. Rather than run a sediment transport model driven by the hydrodynamic model output, innovative manipulation of hydrodynamic model output can provide this insight without having to operate a sediment transport model. Primarily, shear stress at the bed drives sediment transport. With velocity computed at peak ebb/flood as input, shear stresses can be estimated in the following manner: assuming the Manning formula applies locally (a reasonable assumption in working with the time scales of tidal flow which is quasi-steady state), the Manning formula, in American customary units, is given by

$$
V=\frac{1.486}{\mathrm{n}} R^{2 / 3} S^{1 / 2}
$$

where $V$ is the depth-averaged velocity, $\mathrm{n}$ is Manning's $\mathrm{n}, R$ is the hydraulic radius (assumed to be the local water depth), and $S$ is the slope of the energy grade line. By momentum conservation, for steady state flows, the following equation applies

$$
\tau=\rho g R S
$$

where $\tau$ is the shear stress at the bed, $\rho$ is the mass density, $g$ is gravity, $R$ is the hydraulic radius, and $S$ is the slope of the energy grade line. Combining Equations 1 and 2 and eliminating the slope of the energy grade line yields

$$
\tau=\frac{\rho g V^{2}}{\left(\frac{1.486}{\mathrm{n}}\right)^{2} R^{1 / 3}}
$$

In regions of uniform Manning's n, creating plots of shear stress is possible within SMS. Entering Equation 3 in the data calculator yields a scalar data set that SMS can plot (Figure 12). To create vectors of shear stress, one must first assume that the shear stress acts in the direction of the velocity. Next, the velocity vector data set is deconstructed into scalar data sets of velocity direction and magnitude. Finally, the shear stress vector data set is constructed by combining the shear stress magnitude scalar data set and the velocity direction scalar data set. The vectors in Figure 12 are proportional to the shear stress magnitude. 


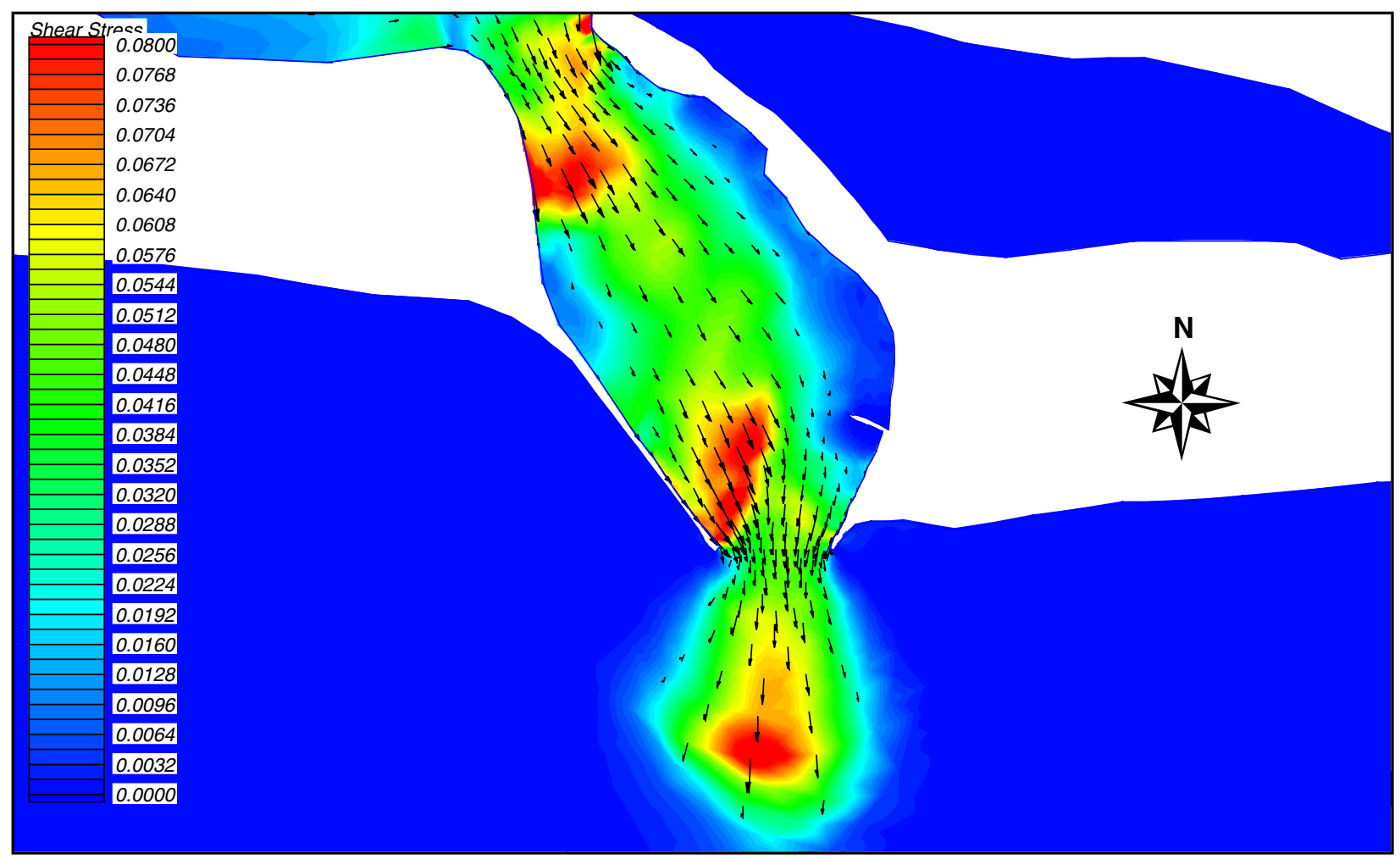

Figure 12. Contours of shear stress overlaid with shear stress vectors (units in lbs/ft ${ }^{2}$ )

Figure 12 clearly shows the regions of increased shear stress (in red) and thus increased sediment transport. The combination of high velocity and shallow water over the west interior shoal in the channel at both the north and south end and over the ebb shoal cause large shear stresses at the bed.

Calculating the sediment transport directly via a total load function produces a more rigorous assessment of sediment transport. Figure 13 shows contours of sediment transport magnitude calculated with the Ackers-White (1973) total load formula. To perform the calculations, velocities computed by the hydrodynamic model were written to an ASCII file. Next, sediment transport was calculated by the following equation:

$$
\frac{Q_{s}}{V d}=\frac{D_{35}}{d}\left(\frac{V}{u_{*}}\right)^{p} C_{1}\left(\frac{F-A}{A}\right)^{m}
$$

where $Q_{s}$ is the time mean sediment transport rate of the sediment, $d$ is the local depth, $u *$ is the friction velocity, and $D_{35}$ is the sediment diameter for which 35 percent of the sediment comprising the bed is finer by weight. The variables $p, m, F$ and $A$ are functions of the flow and sediment parameters as defined subsequently. The parameter $F$ is given by the equation 


$$
F=\left(\frac{V}{2.46 \ln \left(\frac{10 d}{D_{35}}\right)}\right)^{1-p} \frac{u_{*}^{p}}{\left[(s-1) g D_{35}\right]^{1 / 2}}
$$

where $s$ is the sediment specific gravity. In addition, if

$$
D_{*}=\left((s-1) \frac{g}{v^{2}}\right)^{1 / 3} D_{35}
$$

where $v$ is the kinematic viscosity, then for $D *>60$

$$
\begin{aligned}
p & =0 \\
A & =0.17 \\
m & =1.5 \\
C_{1} & =0.025
\end{aligned}
$$

and for $1<D * \leq 60$

$$
\begin{aligned}
p & =1-0.243 \ln D_{*} \\
A & =\frac{0.23}{\sqrt{D_{*}}}+0.14 \\
m & =\frac{9.66}{D_{*}}+1.34 \\
C_{1} & =\exp \left[2.86 \ln D_{*}-0.434\left(\ln D_{*}\right)^{2}-8.13\right]
\end{aligned}
$$

This scalar data set is then read back into SMS to create the contour plot seen in Figure 13. Notably, entering a simpler total load sediment transport formula into the data calculator will create the similar data set. 


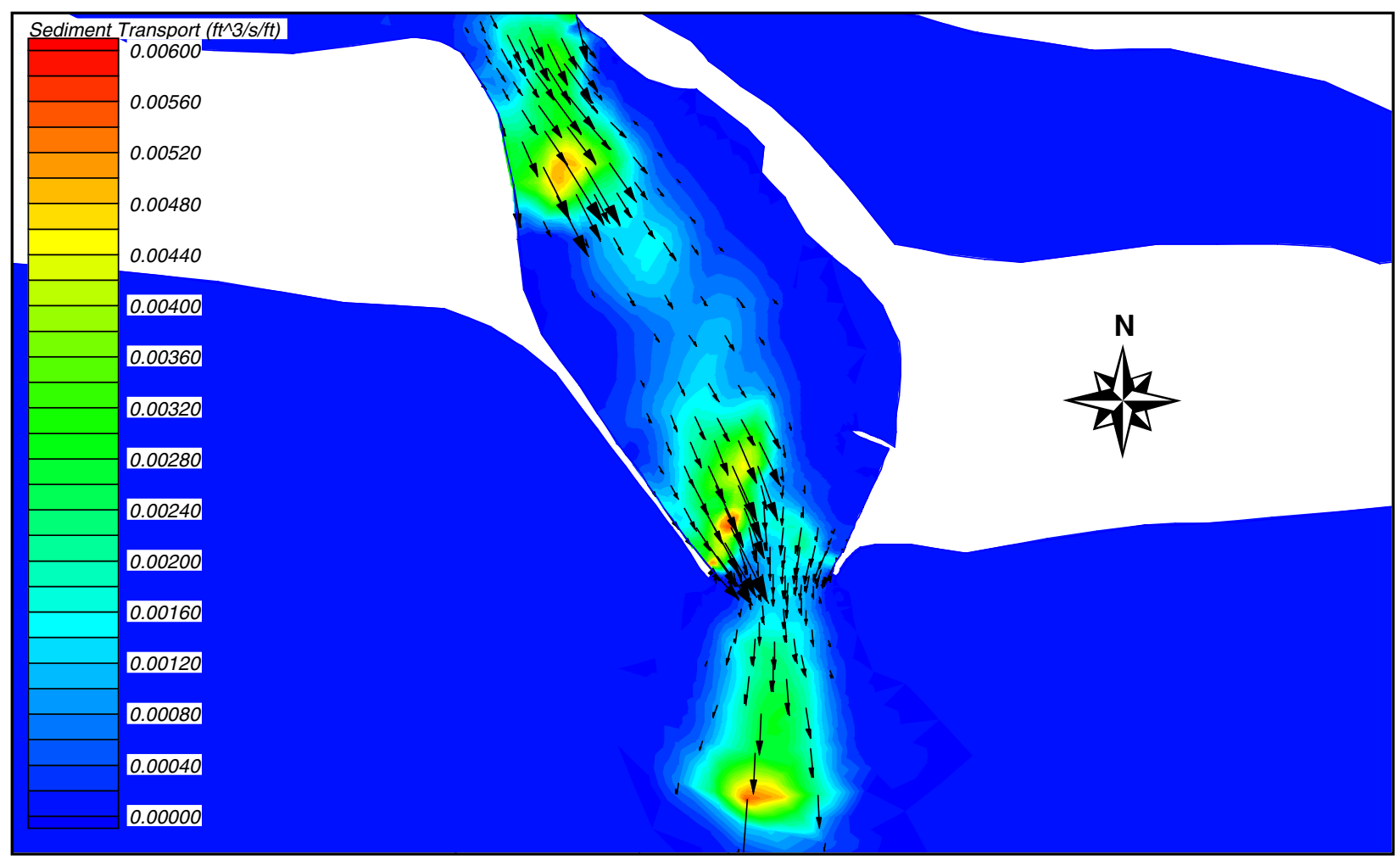

Figure 13. Contours of sediment transport overlaid with sediment transport vectors (units in $\mathrm{ft}^{3} / \mathrm{s} / \mathrm{ft}$ ) (To convert cubic feet per second to cubic meters per second, multiply by 0.02831685 )

The vector data set is constructed in the same manner as the shear stress vector data set. First, one must assume that sediment transport acts in the same direction as the velocity. Next, the velocity vector data set is reduced into velocity-magnitude and velocity-direction scalar data sets. Finally, the velocity direction and sediment transport magnitude scalar data sets are combined to construct the sediment transport vector data set.

Figure 13 makes apparent regions of considerable sediment transport (in red). Again, the plot highlights the same regions as in previous plots -at the north and south extremes of the channel over the western shoal and over the outer bar of the ebb shoal. In interpreting sediment transport plots, one must read the gradients of sediment transport in the direction of sediment transport to infer bed elevation change. For example, as water flows from regions of weak sediment transport to regions of strong sediment transport, scouring of the bed occurs in the region of strong sediment transport because more sediment leaves the area downstream than enters from upstream. Conversely, as water flows from regions of strong sediment transport to regions of weak sediment transport, deposition occurs in the region of weak sediment transport because more sediment enters the area from upstream than leaves downstream. From the figure, the red region over the ebb shoal bar should experience erosion because the sediment-transport gradient increases in the flow direction. South of this point, an area of deposition should occur because the sediment-transport gradient decreases in the flow direction. The net effect is an offshore translation of the ebb shoal bar. 
CONCLUSIONS: This CETN has presented several methods for improving the diagnostic capabilities of plots created from hydrodynamic output with the SMS platform. These visualization methods included techniques for viewing the default output as well as methods for manipulating the output to gain insight into modification of flow through engineered modifications and into the sediment transport driven by the flow.

ADDITIONAL INFORMATION: Questions about this CETN can be addressed to Dr. Mark S. Gosselin (904-731-7040, fax 904-731-9847, e-mail: mgosselin@taylorengineering.com) or to Dr. Nicholas C. Kraus (601-634-2016, Fax 601-634-3080, e-mail: krausn@wes.army.mil)

This CETN should be cited as follows:

Gosselin, M.S., Taylor, R.B., and Craig, K.R. (2000). "Maximizing understanding of model results through graphical displays," ERDC/CHL CETN-IV-27, U.S. Army Engineer Research and Development Center, Vicksburg, MS, http://chl.wes.army.mil/library/publications/cetn/

\section{REFERENCES}

Ackers, P., and White, W. R. (1973). "Sediment transport: new approach and analysis." Proceedings A.S.C.E. Journal of Hydraulics Division, 99(HY11), 2041-2060.

Brigham Young University. (1999). Surface-Water Modeling System reference manual, version 6.0. Brigham Young University, Environmental Modeling Research Laboratory. Provo, UT.

Kraus, N. C. (2000) "Introduction to the Diagnostic Modeling System (DMS)," ERDC/CHL CETN-IV28, U.S. Army Engineer Research and Development Center, Vicksburg, MS, http://chl.wes.army.mil/library/publications/cetn/. 